\title{
Multi-Center Study of Resectable Lung Lesions by Ultra-Deep Sequencing of Targeted Genes in Plasma Cell-Free DNA to Assess Nodule Malignancy and Detect Lung Cancers
}

Muyun Peng ${ }^{1, \#}$, Yuancai Xie ${ }^{2, \#}$, Xiaohua $\mathrm{Li}^{3, \#}$, Youhui Qian" ${ }^{4}$, Xiaonian $\mathrm{Tu}^{3}$, Xumei Yao ${ }^{3}$, Fangsheng Cheng ${ }^{3}$, Feiyue $\mathrm{Xu}^{3}$, Deju Kong ${ }^{3}$, Bing $\mathrm{He}^{5}$, Chaoyu $\mathrm{Liu}^{3}$, Fengjun $\mathrm{Cao}^{6}$, Haoxian Yang ${ }^{7}$, Jiankui He${ }^{8}$, Fenglei $\mathrm{Yu}^{1^{*}}$, Chuanbo $\mathrm{Xu}^{3, *}$, Geng Tian ${ }^{4, *}$

${ }^{1}$ Department of Thoracic Surgery, No.2 Xiangya Hospital, Central South University, Changsha, 410011, China

${ }^{2}$ Thoracic Department, Peking University Shenzhen Hospital, Shenzhen, 518036, China

${ }^{3}$ Vienomics Biotech Co., Ltd., Shenzhen, 518053, China

${ }^{4}$ Department of Oncology, Shenzhen Second People's Hospital, The First Affiliated Hospital of Shenzhen University, Shenzhen, 518035, China

${ }^{5}$ Department of Clinical Pharmacy, University of Michigan, Ann Arbor, MI, USA

${ }^{6}$ Laboratory of Chinese Herbal Pharmacology, Oncology Center, Renmin Hospital, Hubei University of Medicine, Shiyan, 442000, China

${ }^{7}$ Department of Thoracic Surgery, Sun Yat-sen University Cancer Center; State Key Laboratory of Oncology in South China; Collaborative Innovation Center for Cancer Medicine. Guangzhou, China

${ }^{8}$ Department of Biology, South University of Science and Technology of China, Shenzhen, 518055, China

\#Authors contributed equally.

*Corresponding authors: C. Xu, xcb@vienomics.com; F. Yu, yufenglei@csu.edu.cn; G. Tian, tiangeng666@aliyun.com

\begin{abstract}
BACKGROUND Early detection of lung cancer to allow curative treatment remains challenging.

Cell-free circulating tumor DNA (ctDNA) analysis may aid in malignancy assessment and early cancer diagnosis of lung nodules found in screening imagery.

METHODS The multi-center clinical study enrolled 192 patients with operable occupying lung diseases. Plasma ctDNA, white blood cell genomic DNA (gDNA) and tumor tissue gDNA of each
\end{abstract}


patient were analyzed by ultra-deep sequencing to an average of 35,000X of the coding regions of 65 lung cancer-related genes.

RESULTS The cohort consists of a quarter of benign lung diseases and three quarters of cancer patients with all histopathology subtypes. $64 \%$ of the cancer patients is at Stage I. Gene mutations detection in tissue gDNA and plasma ctDNA results in a sensitivity of $91 \%$ and specificity of $88 \%$. When ctDNA assay was used as the test, the sensitivity was $69 \%$ and specificity $96 \%$. As for the lung cancer patients, the assay detected 63\%, 83\%, 94\% and 100\%, for Stage I, II, III and IV, respectively. In a linear discriminant analysis, combination of ctDNA, patient age and a panel of serum biomarkers boosted the overall sensitivity to $80 \%$ at a specificity of $99 \%$. 29 out of the 65 genes harbored mutations in the lung cancer patients with the largest number found in TP53 (30\% plasma and 62\% tumor tissue samples) and EGFR (20\% and 40\%, respectively).

CONCLUSION Plasma ctDNA was analyzed in lung nodule assessment and early cancer detection while an algorithm combining clinical information enhanced the test performance.

\section{Introduction}

Lung cancer is the leading cause of cancer-related deaths, accounting for an estimated 1.6 million deaths each year globally (1). The prognosis of lung cancer is dependent on the stage of diagnosis, with 5-year overall survival rate decreasing dramatically from stage IA (85\%) to stage IV disease $(6 \%)(2,3)$. This makes it clearly a winning strategy to screen and diagnose lung cancer earlier to saving lives (3).

Current method for lung cancer screening is low-dose computed tomography (LDCT) (4). The National Lung Screening Trial (NLST) demonstrated a $20 \%$ reduction in lung cancer mortality for LDCT compared with $X$ ray and a $6.7 \%$ all-cause mortality reduction (5). However, the imagery technique often results in indeterminate nodules (6) and the false positive results lead to unnecessary invasive diagnostic procedures and increased deaths from avoidable surgeries (7).

Traditionally, biopsy is used to determine malignancy of lung nodules. This approach has significant limitations as being difficult or even impossible. Molecular tests using pervasive 
biofluid samples, so called liquid biopsy, are promising and urgently needed in the thoracic clinic, as demonstrated in thyroid nodules assessment where a number of molecular tests are available (8). In pulmonary nodule malignancy assessment, there is also the report of blood proteomics biomarkers by the PANOPTIC team (9).

In theory, ctDNA is exquisitely specific for an individual's tumor as by definition somatic mutations are identified by their presence in tumor DNA and absence in matched normal DNA. This bypasses the issues related to the false-positivity encountered with other biomarkers, such as protein biomarkers. This specific and promising early detection method has garnered tremendous attention for cancer in general and lung in particular $(10,11)$. The growing interest has drawn attention from international societies such as IASLC that has issued statement regarding liquid biopsy in the management of non-small cell lung cancer (NSCLC) (12).

Early detection of cancer by blood test was shown possible even with microscopic tumor proceeding radiography (13). Most recently, a preliminary analysis of early to mid-stage (stage IIII) lung cancer patients as part of the Circulating Cancer Genome Atlas (CCGA) pan-cancer study was reported (14).

In addition to mutation analysis, ctDNA epigenetics has also been evaluated and researched for early cancer detection. The studies examined DNA methylation regulation (15) or the hypermethylation of the promoter regions of genes (16) for the potential biomarkers of lung cancer detection.

In this study, we report a multi-center clinical trial result on ultra-deep sequencing in patients that undergo surgical resection either benign nodules or with different stages of lung cancer.

\section{Patients \& Methods}

\section{Clinical Centers and Patients}

The clinical trial (ClinicalTrial.gov NCT03081741) at four Tier A hospitals in China recruited patients with lung occupying diseases to be treated by surgery. Patients with benign lung nodules or cancers of Stage I to III planned for surgical resection were eligible for inclusion (Table S1). After informed consenting, 
biological samples (two tubes of $10 \mathrm{~mL}$ peripheral blood prior to surgery collected in a cell-free DNA (cfDNA) BCT blood collection tube (Streck, Omaha, US) and 10 slides of formalin fixed paraffin embedded (FFPE) tissues from surgery), and related clinical data including serum biomarkers were collected.

\section{Sequencing Analysis}

The above collected DNA samples were analyzed by our proprietary Sec-Seq technique as described previously (17). Briefly, blood sample were processed to separate the plasma from blood cells by centrifugation. cfDNA, gDNA of WBC and FFPE were extracted using QIAamp Circulating Nucleic Aid Kit, DNA mini kit, DNA FFPE Tissue kit, respectively (Qiagen, Hilden, Germany). The concentration of extracted DNA was measured using Qubit 3.0 dsDNA high-sensitivity assay (Life Technologies, Carlsbad, CA).

Capture probes were designed for 65 cancer-associated genes covering $241 \mathrm{~kb}$ genomic regions (Table S2) and synthesized by IDT (MI, USA). Indexed libraries were constructed using KAPA HyperPlus Kit (KK8514). Barcoding was employed to reduce noise. Post-capture multiplexed libraries were amplified with Illumina backbone primers for 16 cycles of PCR using 1× KAPA HiFi Hot Start Ready Mix and sequenced on Illumina NovaSeq platform (Illumina, CA, USA) at Novogen (Nanjing, China).

Horizon's Partners Spike-in control (Horizon, Cambridge, UK) was used in a serial dilution (from 0.0005 to 1) using the wild-type reference genome and the provided reference standard. Reference variants include EGFR (L858R, T790M), KRAS (G12D), NRAS (Q61K, A59T) and PIK3CA (E545K), or NA12878 (12 mutations) and NA24385 (29 mutations).

\section{Bioinformatics Data Analysis}

Reads with quality score $<30$ or havinh $>5 \%$ of positions differ from the rest of the reads targeting the same region were removed. The results were then mapped to the human reference genome (hg19) using BWA (v0.7.15-r1140). We used the start mapping positions, the length and the dual barcode on both side of merged paired-end fragments to form reads groups amplified from every primary cfDNA molecules and to identify incorrect base produced due to PCR errors.

Variant calling for single nucleotide variation (SNV) or insertion/deletion (INDEL) was performed using samtools mpileup tool (v1.3.1). For ctDNA samples, a variant was selected as a candidate somatic mutation when (1) two distinct paired reads (each redundantly sequenced at least three times) contained the mutation, (2) effective reads depth >500 (captured primary cfDNA molecules $>500$ ) and (3) the corresponding allele frequency in WBC is less than $1 \%$. 


\section{Mutation Annotation and Classification}

The variants were called by SnpEff (v4.3o), and annotated by COSMIC (v85), ExAc, ClinVar, and 1000 Genome. The following variants were eliminated: (1) intergenomic or intronic (except for splicing junction); (2) synonymous; (3) variant allele frequency (VAF) $<0.2 \%$ in ctDNA or $<1 \%$ in FFPE samples. Previously reported and confirmed pathogenic mutations in the clinical samples of lung cancer of all human races and ethnicities will be considered as lung cancer related.

\section{Statistical Methods}

Data were summarized using descriptive statistics. Fisher's exact test was used to compare any two subgroups. Wilcoxon rank sum test was used to compare median age between any two subgroups (Stage I, II, III) or mutant groups (mutant vs wild type).

Linear discriminant analysis (LDA) was performed on improving mutation analysis. The model considers the age of the patients, ctDNA mutations, and the serum biomarkers. It was developed using the 10 fold cross validation by dividing the samples into training and validation subsets. The test sensitivity and specificity was calculated, and AUROC was plotted.

\section{Results}

\section{Patient Demographic and Clinical Analysis}

In total, 192 patients with pulmonary space occupying lesions (136 malignant and 56 benign) pathologically diagnosed and surgically treated were included in this analysis. These patients were recruited from 4 clinical sites: Xiangya No.2 Hospital in Changsha, Hunan Province, Beijing University Shenzhen Hospital, Shenzhen, Huizhou People's Hospital, Huizhou, and No.2 People's Hospital in Shenzhen, Guangdong Province. Though excluded but due to delayed pathological determination, three Stage IV patients were enrolled and hence analyzed.

The average age is 56.5 (range 26-79) years and male proportion is $59 \%$. These numbers are 50.1 (26-73) years and 55\% for the benign group, 59.1 (27-79) years and 60\% for lung cancer group with a statistically significant difference. No difference was found between the two groups for smoke status (32\% vs. $35 \%$ ) and family history (7\% vs. $8 \%$ ) (Table 1$)$. 
For the benign lesions, the most common diseases diagnosed are pneumonia $(n=14,25 \%)$, tuberculosis ( $n=12,21 \%)$, pulmonary fibrosis $(n=4,7 \%)$, and necrosis granuloma $(n=3,5 \%)$.

Lung cancer distribution was 87, 29, and 17 in Stages I, II and III, respectively. The average size of the nodules for lung cancer patients is 2.9 (range $0.5-9.0$ ) cm, and for each subgroup: (1) Stage I: $2.2(0.5$ - 4.0) cm; (2) Stage II: 3.8 (1.0 - 7.0) cm; and (3) Stage III: 5.0 (1.3 - 9.0) cm (Table 2). The average size of the nodules in the benign group is $2.3(0.3-6.0) \mathrm{cm}$ which is statistically smaller than that of the malignant group.

All of the patients are with solitary pulmonary nodules, except for two patients who had two nodules each that are malignant.

\section{Genetic Profiling and Mutation Burden}

For each patient, three biospecimen samples including plasma ctDNA, WBC and FFPE tumor tissue were sequenced. For ctDNA, the average sequencing depth is 35000 with 1350 unique reads after deduplication.

In total, 312 occurrences and 274 unique somatic mutations were found in 29 genes from either plasma ctDNA or tissue DNA in 120 cancer and 5 benign cases. No mutations were found in 14 lung cancers in either blood or tissue samples.

In the benign lesions, 2 out of 56 patients (3.6\%) had 4 non-driver gene mutations in ctDNA, and 3 patients (5.4\%) had 2 non-driver mutations in FFPE samples.

Among the lung cancer patients, 88\% (120 out of 136 patients) were found to harbor at least one mutation in ctDNA or tumor tissue. When analyzed by stage of cancer, the class, that is whether driver or non-driver mutation, and number of mutations increase as the stage advances (Figure 1).

Mutations were found in 9 known lung cancer genes of ALK, BRAF, EGFR, HER2, KRAS, MET, NRAS, PIK3CA, and ROS1 (Fig. 2) out of the 12 genes defined as drivers for lung cancer (Ref. 18). The most commonly mutated genes in the lung cancer patients are TP53 (44\%) and EGFR (35\%) (Figure S1). 
The most commonly occurred mutation is EGFR L858R (found in 24 samples) followed by EGFR exon 19 deletion (in 15 samples). The largest number of mutations was found in a patient who harbored 15 mutations.

\section{Concordance between ctDNA and Tumor Tissue}

Concordance is defined as at least one gene mutation is the same in both the plasma ctDNA and the FFPE tumor tissue gDNA of a patient. When completely no mutations were found in both the blood ctDNA and tissue gDNA, it is also considered as concordant of the two samples.

Among the 136 malignant cases, the overall concordance rate is $37 \%$. Among the concordant patients, 35 of them share at least one mutation in blood and tissue samples, and 15 of them had no mutations in either the ctDNA or tissue samples. Concordance was higher in the driver genes, $46 \%$. The shared mutation rate increases as the stage of the cancer advances: $32 \%, 78 \%, 50 \%$, and $67 \%$ at Stage I, II, III, and IV, respectively (Figure S2).

\section{Comparison with Serum Biomarkers}

A panel of six serum protein tumor biomarkers was also analyzed which has a sensitivity of $51 \%$ with a specificity of $83 \%$ (Table 3). These markers include NSE, CYFRA 21-1, CEA, ProGRP, CA-125, and SCC. When the most sensitive marker of CYFRA 21-1 was considered alone, the sensitivity was merely $25 \%$ at a specificity of $95 \%$ (Table 3 ).

In comparison, the profiling by ctDNA showed a higher sensitivity in detecting lung cancer. The sensitivity increases as the stage advances and ctDNA outperform the serum biomarkers in all stages (Figure 3).

\section{Malignancy Assessment and Lung Cancer Tests}

The mutation profiling classifies the lung nodules from benign to malignant (Figure 1). The benign samples harbor very little to no mutations. The cancer patients have increasing level of mutations both in terms of category and numbers as stage progresses. From Stage I to IV, there are more mutations found and they are more often in driver genes. 
For lung cancer detection, the overall sensitivity of plasma ctDNA was $68 \%$ at the specificity of 96\%. Slightly higher detection rate was observed in tissue DNA which reached 78\% (Table 3). According to cancer stages, the sensitivity rate is $60 \%, 69 \%, 94 \%$, and $100 \%$ for Stage I, II, III, and IV, respectively (Fig. 3).

We further conducted a linear discriminant analysis where patient age, smoking status, and serum protein markers were considered (Figure S3). The combined model of ctDNA mutations and serum biomarkers improved the sensitivity and specificity to $80 \%$ and $99 \%$ respectively (Table 3).

\section{Discussion}

Lung cancer screening recommended by the guidelines targets populations at high risk of developing lung cancer, such as patients aged above 55 years, heavy smokers and having chronic obstructive pulmonary disease (COPD) and with family history of lung cancer. In general, by definition of $50-500 \mathrm{~mm}^{3}$ (19), screening by imagery techniques could result in about 30-40\% indeterminate nodules that need to be further evaluated. In our cohort, we have $>60 \%$ nodules in this size range making it an urgent need for malignancy assessment.

Our patient population drawn from major hospitals in southern and central China has a median age of 56.5 years, just above the threshold for lung cancer screening. The clinical outcome confirmed the age as a risk factor for lung cancer: the median age of the patients with benign nodules (50.1 years) was about ten years younger than those having lung cancer (median age of 59.1 years).

Smoking status and family history were not significantly different between the benign and the malignant cases in our cohort. One possible explanation could be that the impact of smoking should be considered more broadly than tabaco smoking alone. For example, the time spent on Chinese style cooking could be a potential risk. Unfortunately, no such data were collected. As a proxy, however, the gender distribution difference could be explored since female usually spends 
more time in the kitchen. While we have more male patients in the cancer group (about 60\%), the opposite is true for the benign group which has less (about 55\%).

It is reported that tumor burden of lung cancer corresponds to its size (20). Our data confirms that the size of the nodule relates to malignancy and progression. The average tumor size increases from $22 \mathrm{~mm}$ in Stage I to $38 \mathrm{~mm}$ in Stage II and to $50 \mathrm{~mm}$ in Stage III (Table 2). However, there is no significant difference between a benign nodule and that of the Stage I cancer (Table 1 and 2).

The concordance rate between tissue and corresponding plasma ctDNA also reflects the challenge of early cancer liquid biopsy. Our study is heavy in Stage I patients (64\%, Table 2) which has a rate of $32 \%$ and causes the overall rate of $36 \%$. The rate increases as stage advances - up to the highest of $78 \%$ and the average of Stage I-III is 53\%. In CCGA, a set of 73 early to mid-stage (stage I-III) lung cancer samples showed a similar rate of $59 \%(\mathrm{Cl}=47-70 \%)(13)$. In another very small study of 31 paired lung cancer tissues and plasma DNA samples with 10,000-fold ctDNA sequencing depth, the concordance of mutation between tumor tissue DNA and ctDNA was merely $3.9 \%$ (21). Ours is more like CCGA in that we both sequenced ctDNA to the depth $>40,000 \mathrm{X}$. Another meta-analysis has also put the pooled sensitivity in the range of $60 \%$ 's (22).

The gene mutations shared between the plasma ctDNA and the FFPE tumor tissue increase as the lung cancer stage advances. This is in alignment with the previous report that Stage IV tumor has the highest concordance (23), as well as that as the tumor is getting larger, the amount of DNA fragments it sheds into the blood stream will increase $(20,24)$.

Although somatic mutations showed strong feasibility of detecting malignancy and staging the cancer by plasma ctDNA, there more factors to be considered. Therefore, combining clinical and genomic features improved the test performance as shown by our LDA modeling (Table 3). Integrated classifiers have also been explored in terms of plasma protein biomarkers such as the ones tested by some panel components in our cohort (25).

Liquid biopsy starts moving into cancer clinics in therapy selection (26, reviewed in 27). For early detection of lung cancer, one study involving 60 NSCLC patients, 40 COPD patients and 40 healthy controls showed that serum cfDNA concentrations and integrity may be valuable (27). Another 
study identified 17 miRNA species in the exosomes of the blood that are differentially expressed in cancer (both NSCLC and SCLC) and controls (27). The potential use of ctDNA for early detection of other cancers has also been reported $(29,30)$.

There are, however, a number of limitation and challenges. First of all, the sample sizes of the early detection studies are usually small especially the number of healthy controls. Second, ctDNA amount correlates with cancer stage (31). Therefore, the consensus is that ultra-deep sequencing of $40,000 \mathrm{X}$ is required to detect the low frequency mutations in the $10 \mathrm{~mL}$ blood. Finally, the less than expected driver mutation concordance between ctDNA and tumor DNA may reflect genetic heterogeneity and indicate tumor evolution (32) suggesting that other types of genes and mutations should be considered as well.

Some biomarkers for lung cancer in blood can be present in patients without identifiable radiological nodules and thus precede the apparition of these nodules. We are continuing with long term follow-up to study these biomarkers. We also continue working on technical improvement of the assay as well as the clinical validation.

\section{Statements}

\section{Contributorship}

$M P, Y X, Y Q, F C, H Y, F Y, G T$ participated in patient recruitment; $F C, C L, C X$ participated in clinical trial and patient sample management; $X L, X Y, F X$ participated in genomic sequencing; $X T, D K, B H, J H, C X$ participated in bioinformatics data analysis; and CX, XL, FY, GT participated in manuscript writing and review.

Funding

This work was supported by Innovation Fund of Shenzhen China (Grant No:

CKCY2016082916544973 and Grant No: CYZZ20170406170950746); Technological Innovation

Research Program of Shenzhen China (Grant No: JSGG20160428090301587 and Grant No: 
JSGG201704141042216477); State Key Research Program of China (Grant No:

2016YFA0501604); the Young Scientist Innovation Team Project of Hubei Colleges (Grant No: T201510); the Key Project of Health and Family Planning Commission of Hubei Province (Grant No: WJ2017Z023), Science technology and innovation committee of Shenzhen for research projects (JSGG20160428090301587), Scientific Research Project of Shenzhen Health and Family Planning System (SZLY2017008/SZLY2018020).

Competing Interests

The authors have no competing interest in this publication.

\section{Acknowledgement}

The authors would like to thank the nurses in the Department of Thoracic Surgery, No.2 Xiangya Hospital, Thoracic Department, Peking University Shenzhen Hospital, Department of Oncology, Shenzhen Second People's Hospital, Oncology Center and Department of Thoracic Surgery, Sun Yat-sen University Cancer Center for their work in enrolling the patients, collecting biospecimen samples, and related clinical data. We would like to thank the laboratory staff at Vienomics for processing the samples and conducting the sequencing procedures. Finally but not the least, we would like to express our highest appreciation of the participation and contribution of our patients in the study.

\section{References}

1. Ferlay J, Soerjomataram I, Dikshit R, et al. Cancer incidence and mortality worldwide: sources, methods and major patterns in GLOBOCAN 2012. Int J Cancer 2015;136:E359-86.

2. Goldstraw P, Chansky K, Crowley J, et al. The IASLC Lung Cancer Staging Project: Proposals for Revision of the TNM Stage Groupings in the Forthcoming (Eighth) Edition of the TNM Classification for Lung Cancer. J Thorac Oncol 2016;11:39-51.

3. Henschke $\mathrm{Cl}$, Yankelevitz DF, Libby DM, et al. Survival of patients with stage I lung cancer detected on CT screening. N Engl J Med 2006;355:1763-71. 
4. Patz EF, Goodman PC, Bepler G. Screening for lung cancer. N Engl J Med 2000;343:1627-33.

5. National Lung Screening Trial Research Team, Aberle DR, Adams AM, et al. Reduced lungcancer mortality with low-dose computed tomographic screening. N Engl J Med 2011;365:395409.

6. Kakinuma, R. et al. Evaluation of the $95 \%$ limits of agreement of the volumes of 5 -year clinically stable solid nodules for the development of a follow-up system for indeterminate solid nodules in CT lung cancer screening. J Thorac Dis 2018;10(1):175-189.

7. Tammemagi MC, Katki HA, Hocking WG, et al. Selection criteria for lung-cancer screening. N Engl J Med 2013;368:728-36.

8. Nicholson KJ, Yip L. An update on the status of molecular testing for the indeterminate thyroid nodule and risk stratification of differentiated thyroid cancer. Curr Opin Oncol. 2018 Jan;30(1):8-15.

9. Silvestri GA, Tanner NT, Kearney P, et al for PANOPTIC Trial Team. Assessment of Plasma Proteomics Biomarker's Ability to Distinguish Benign From Malignant Lung Nodules: Results of the PANOPTIC (Pulmonary Nodule Plasma Proteomic Classifier) Trial. Chest. 2018 Sep;154(3):491-500.

10. Matikas A, Syrigos KN, Agelaki S. Circulating Biomarkers in Non-Small-Cell Lung Cancer: Current Status and Future Challenges. Clin Lung Cancer 2016;17:507-16.

11. Levy B, Hu ZI, Cordova KN, et al. Clinical Utility of Liquid Diagnostic Platforms in Non-Small Cell Lung Cancer. Oncologist 2016;21:1121-30.

12. Rolfo C, Mack PC, Scagliotti GV, et al. IASLC Statement Paper: Liquid Biopsy for Advanced Non-Small Cell Lung Cancer (NSCLC). J Thorac Oncol. 2018 Jun 6. pii: S1556-0864(18)30680-4.

13. Abbosh C, Birkbak NJ, Wilson GA, et al. Phylogenetic ctDNA analysis depicts early-stage lung cancer evolution. Nature. 2017 Apr 26;545(7655):446-451.

14. Klein EA, Hubbell E, Maddala T, et al. Development of a comprehensive cell-free DNA (cfDNA) assay for early detection of multiple tumor types: The Circulating Cell-free Genome Atlas (CCGA) study. Abstract\#161733. ASCO Conference. June 4, 2018. Chicago, IL, USA.

15. Greenberg AK, Rimal B, Felner K, et al. S-adenosylmethionine as a biomarker for the early detection of lung cancer. Chest. 2007 Oct;132(4):1247-52.

16. Ostrow KL, Hoque MO, Loyo M, et al. Molecular analysis of plasma DNA for the early detection of lung cancer by quantitative methylation-specific PCR. Clin Cancer Res. 2010 Jul 1;16(13):3463-72.

17. Xia L, Li Z, Zhou B, et al. Statistical analysis of mutant allele frequency level of circulating cell-free DNA and blood cells in healthy individuals. Sci Rep. 2017 Aug 8;7(1):7526. 
18. Lovly, C., L. Horn, W. Pao. 2018. Molecular Profiling of Lung Cancer. My Cancer Genome https://www.mycancergenome.org/content/disease/lung-cancer/ (Updated March 16). Last accessed on 13 July 2018

19. Kakinuma R, Muramatsu Y, Yamamichi J, Gomi S, Oubel E, Moriyama N. Evaluation of the 95\% limits of agreement of the volumes of 5-year clinically stable solid nodules for the development of a follow-up system for indeterminate solid nodules in CT lung cancer screening. J Thorac Dis. 2018 Jan;10(1):175-189.

20. Jahr S Hentze H Englisch S, etal.: DNA fragments in the blood plasma of cancer patients: Quantitations and evidence for their origin from apoptotic and necrotic cells Cancer Res 61:1659-1665,2001

21. Ye M, Li S, Huang W, et al. Comprehensive targeted super-deep next generation sequencing enhances differential diagnosis of solitary pulmonary nodules. J Thorac Dis. 2018 Apr;10(Suppl 7):S820-S829.

22. Luo J, Shen L, Zheng D. Diagnostic value of circulating free DNA for the detection of EGFR mutation status in NSCLC: A systematic review and meta-analysis. Sci Rep 2014;4:6269.

23. Douillard JY, Ostoros G, CoboMet al. Gefitinib treatment in EGFR mutated Caucasian NSCLC: Circulating-free tumor DNA as a surrogate for determination of EGFR status. J Thorac Oncol 2014;9:1345-1353.

24. Diehl F Schmidt K Choti MA, et al.: Circulating mutant DNA to assess tumor dynamics Nat Med 14:985-990,2008

25. Guida F, Sun N, Bantis LE, et al for Integrative Analysis of Lung Cancer Etiology and Risk (INTEGRAL) Consortium for Early Detection of Lung Cancer. Assessment of Lung Cancer Risk on the Basis of a Biomarker Panel of Circulating Proteins. JAMA Oncol. 2018 Jul 12:e182078. doi: 10.1001/jamaoncol.2018.2078.

26. Cabanero M, Tsao MS. Circulating tumour DNA in EGFR-mutant non-small-cell lung cancer. Curr Oncol. 2018 Jun;25(Suppl 1):S38-S44.

27. Hu Y, Alden RS, Odegaard JI, et al. Discrimination of Germline EGFR T790M Mutations in Plasma Cell-Free DNA Allows Study of Prevalence Across 31,414 Cancer Patients. Clin Cancer Res. 2017 Dec 1;23(23):7351-7359.

28. Soliman SE, Alhanafy AM, Habib MSE, et al. Serum circulating cell free DNA as potential diagnostic and prognostic biomarker in non small cell lung cancer. Biochem Biophys Rep. 2018 Jun 30;15:45-51.

29, Poroyko V, Mirzapoiazova T, Nam A, et al. Exosomal miRNAs species in the blood of small cell and non-small cell lung cancer patients. Oncotarget. 2018 Apr 13;9(28):19793-19806. 
30. Leary RJ Sausen M Kinde I, et al.: Detection of chromosomal alterations in the circulation of cancer patients with whole-genome sequencing Sci Transl Med 4:162ra154,2012

31. Schwarzenbach H Müller V Milde-Langosch K, etal.: Evaluation of cell-free tumour DNA and RNA in patients with breast cancer and benign breast disease Mol Biosyst 7:2848-2854,2011

32. DiehI F, Li M, Dressman D, etal.: Detection and quantification of mutations in the plasma of patients with colorectal tumors Proc Natl Acad Sci U S A 102:16368-16373,2005

33. Pezzuto A, Manicone M, Scaini MC, Ricci A, Mariotta S, Zamarchi R, Rossi E. What information could the main actors of liquid biopsy provide? -a representative case of non-small cell lung cancer (NSCLC). J Thorac Dis. 2018 Jul;10(7):E570-E576. 
Table 1. Patient Demographics

\begin{tabular}{|c|c|c|c|}
\hline & Non-Cancer & Cancer & Total \\
\hline Patients & $n=56$ & $n=136$ & $n=192$ \\
\hline \multicolumn{4}{|l|}{ Sex } \\
\hline Male & $31(55.4 \%)$ & $82(60.3 \%)$ & $113(58.9 \%)$ \\
\hline Female & $25(44.6 \%)$ & $54(39.7 \%)$ & $79(41.1 \%)$ \\
\hline Average Age (Range) & $50.1(26-73)$ & $59.1(27-79)$ & $56.5(26-79)$ \\
\hline \multicolumn{4}{|l|}{ Smoking History } \\
\hline Yes & $18(32.1 \%)$ & $48(35.3 \%)$ & $66(34.4 \%)$ \\
\hline No & $38(67.9 \%)$ & $88(64.7 \%)$ & $126(65.6 \%)$ \\
\hline \multicolumn{4}{|l|}{ Family History } \\
\hline Yes & $4(7.1 \%)$ & $11(8.1 \%)$ & $15(7.8 \%)$ \\
\hline No & $52(92.9 \%)$ & $125(91.9 \%)$ & $177(92.2 \%)$ \\
\hline \multicolumn{4}{|c|}{ Lung Nodule Size (mm, Avg. Max } \\
\hline Dimension) & 23.3 & 29.4 & 27.6 \\
\hline
\end{tabular}


bioRxiv preprint doi: https://doi.org/10.1101/453803; this version posted October 26, 2018. The copyright holder for this preprint (which was not certified by peer review) is the author/funder, who has granted bioRxiv a license to display the preprint in perpetuity. It is made available under aCC-BY-NC-ND 4.0 International license.

Table 2. Lung Cancer Histological Diagnosis and Stages

\section{Number of Pts (percentage)}

Pathological Diagnosis

Adenocarcinoma $100(73.5 \%)$

Squameous Carcinoma $28(20.6 \%)$

Small Cell Lung Cancer $1(0.7 \%)$

Others $7(5.2 \%)$

Clinical Stage

I

II

III

IV
$87(64.0 \%)$

$29(21.3 \%)$

$17(12.5 \%)$

$3(2.2 \%)$
Average Nodule

Size (mm, largest

dimension)
24.3

49.8

18

22.3

22

39

52.1

21 
bioRxiv preprint doi: https://doi.org/10.1101/453803; this version posted October 26, 2018. The copyright holder for this preprint (which was not certified by peer review) is the author/funder, who has granted bioRxiv a license to display the preprint in perpetuity. It is made available under aCC-BY-NC-ND 4.0 International license.

Table 3. Lung nodule malignancy prediction diagnostic test Performance

\begin{tabular}{|c|c|c|c|}
\hline Description & No. of Pts & Sensitivity & Specificity \\
\hline Plasma ctDNA and FFPE & 192 & $91 \%$ & $88 \%$ \\
\hline ctDNA mutation (average) & 192 & $68 \%$ & $96 \%$ \\
\hline Stage I lung cancer & 87 & $63 \%$ & $96 \%$ \\
\hline Stage II lung cancer & 29 & $83 \%$ & $96 \%$ \\
\hline Stage III lung cancer & 17 & $94 \%$ & $96 \%$ \\
\hline Stage IV lung cancer & 3 & $100 \%$ & $96 \%$ \\
\hline tissue mutation (average) & 192 & $81 \%$ & $95 \%$ \\
\hline Panel of six serum biomarkers (BMs) & 175 & $51 \%$ & $83 \%$ \\
\hline LDA model of ctDNA and BMs & 174 & $80 \%$ & $99 \%$ \\
\hline
\end{tabular}


bioRxiv preprint doi: https://doi org/10.1101/453803. this version posted October 26, 2018. The copyright holder for this preprint (which was not certified by peer review) is the author/funder, who has granted bioRxiv a license to display the preprint in perpetuity. It is made available under aCC-BY-NC-ND 4.0 International license. 
Figure 1. Lung Cancer Gene Mutation Burden in Benign and Various Stages of Patients

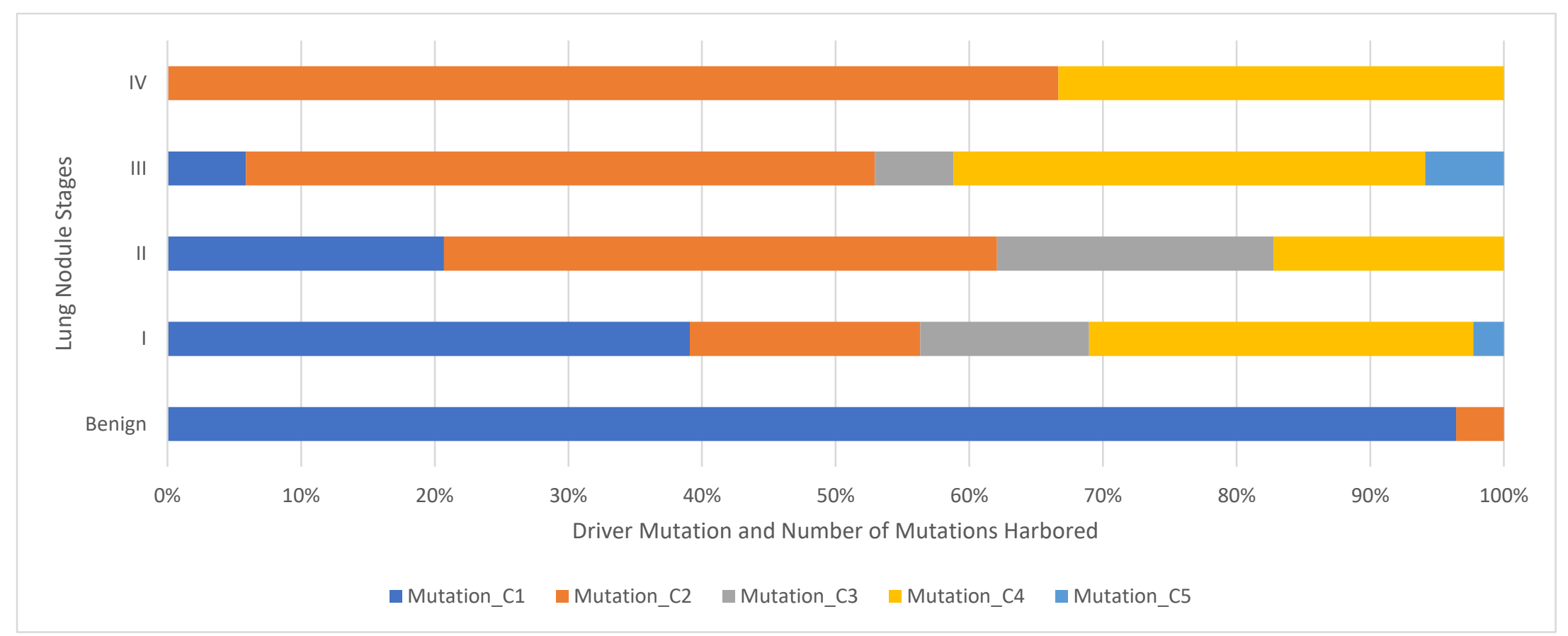

Notes: Mutation types: C1. No mutation found; C2. 1 non-driver mutation found; C3. 1 driver mutation found; C4. Both driver mutation and non-driver mutation found; C5. More than 1 non-driver mutation found. 
bioRxiv preprint doi: https://doi.org/10.1101/453803; this version posted October 26,2018 . The copyright holder for this preprint (which was not certified by peer review) is the author/funder, who has granted bioRxiv a license to display the preprint in perpetuity. It is made available under aCC-BY-NC-ND 4.0 International license.

Figure 2. Driver Mutation Distribution in Lung Cancer Patients

A. Plasma ctDNA

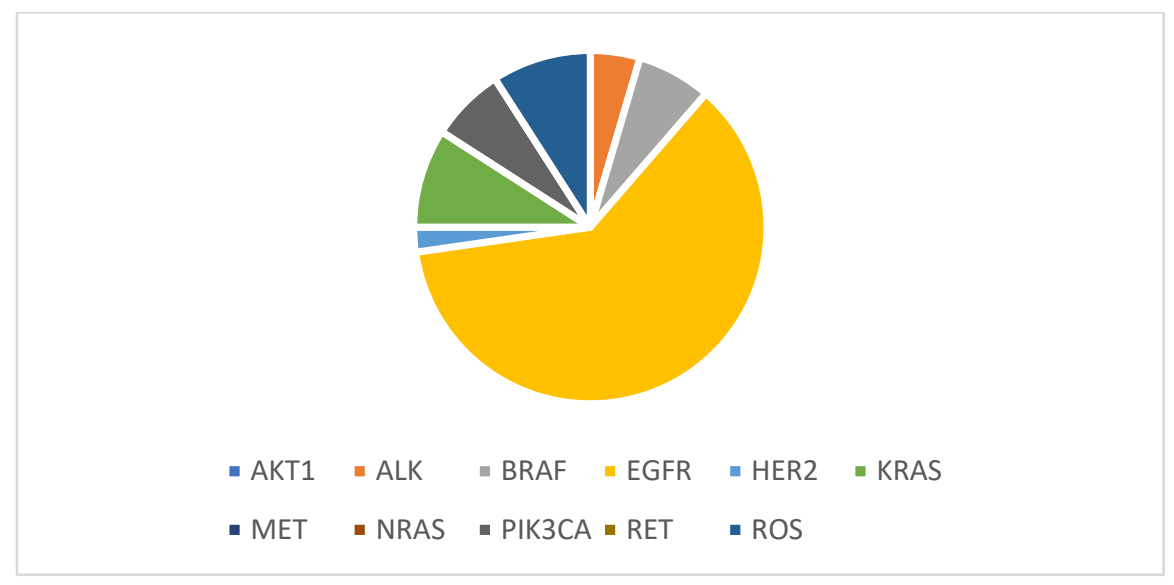

B. FFPE gDNA

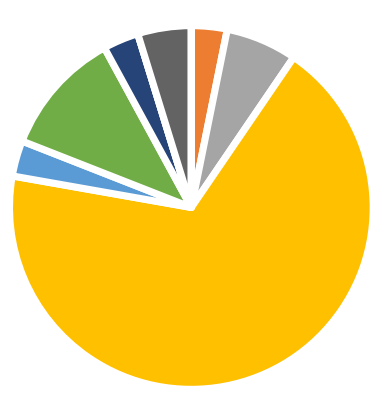

- AKT1 - ALK $\because \mathrm{BRAF}=\mathrm{EGFR}=\mathrm{HER2}=\mathrm{KRAS}$

- MET - NRAS - PIK3CA - RET - ROS 
Figure 3. Lung Cancer Detection Rate by ctDNA, Serum Biomarkers and LDA Model per Stages

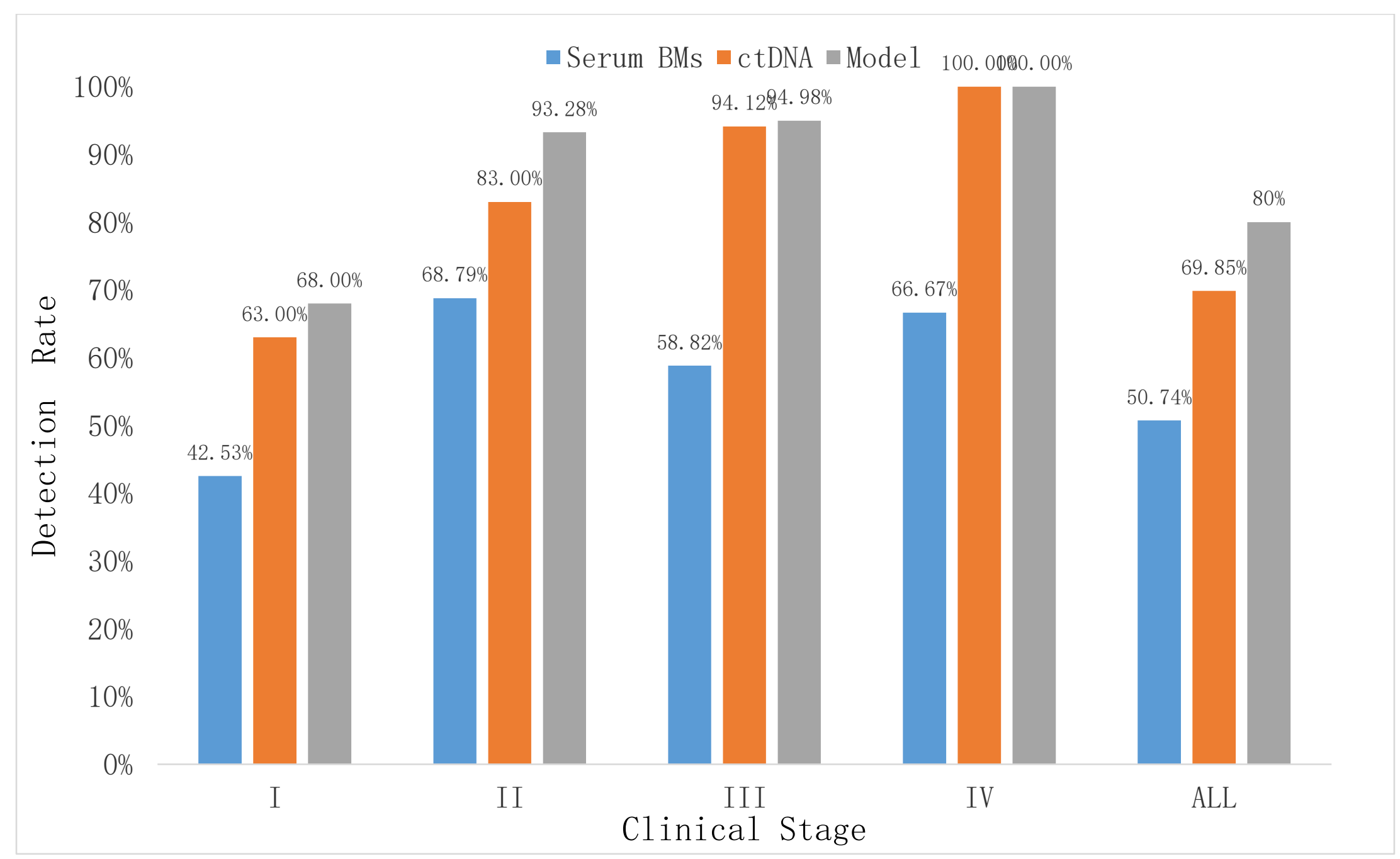

Note: Model = LDA (Linear Discriminant Analysis) model . 